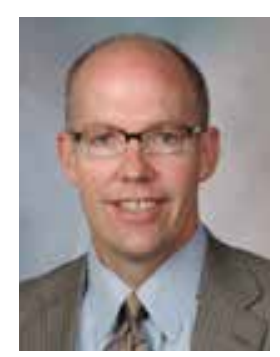

\title{
GENETICS OF LONG QT SYNDROME
}

\author{
David J. Tester, B.S.; Michael J. Ackerman, M.D., Ph.D.
}

Mayo Clinic, Rochester, Minnesota

M.J. Ackerman, M.D., PhD.

\begin{abstract}
Long QT syndrome (LQTS) is a potentially life-threatening cardiac arrhythmia characterized by delayed myocardial repolarization that produces QT prolongation and increased risk for torsades des pointes (TdP)-triggered syncope, seizures, and sudden cardiac death (SCD) in an otherwise healthy young individual with a structurally normal heart. Currently, there are three major LQTS genes (KCNQ1, KCNH2, and SCN5A) that account for approximately 75\% of the disorder. For the major LQTS genotypes, genotype-phenotype correlations have yielded gene-specific arrhythmogenic triggers, electrocardiogram (ECG) patterns, response to therapies, and intragenic and increasingly mutation-specific risk stratification. The 10 minor LQTS-susceptibility genes collectively account for less than 5\% of LQTS cases. In addition, three atypical LQTS or multisystem syndromic disorders that have been associated with QT prolongation have been described, including ankyrin-B syndrome, Anderson-Tawil syndrome (ATS), and Timothy syndrome (TS). Genetic testing for LQTS is recommended in patients with either a strong clinical index of suspicion or persistent QT prolongation despite their asymptomatic state. However, genetic test results must be interpreted carefully.
\end{abstract}

\section{Introduction}

With an estimated incidence as high as 1 in 2,000 persons, congenital LQTS is characterized by delayed repolarization of the ventricular myocardium, QT prolongation (QTc $>480 \mathrm{~ms}$ as the 50th percentile among LQTS cohorts), and increased risk for torsades des pointes (TdP)-mediated syncope, seizures, and sudden cardiac death (SCD) in an otherwise healthy young individual with a structurally normal heart. ${ }^{1}$ While LQTS is rarely inherited recessively and characterized by a severe cardiac phenotype and sensorineural hearing loss, ${ }^{2}$ it is typically inherited as an autosomal-dominant trait. ${ }^{3}$ Sporadic de novo germline mutations may account for nearly $5 \%$ to $10 \%$ of LQTS. At the molecular level, LQTS comprises a collection of several distinct cardiac channelopathies. To date, there are three major LQTS genes and 10 minor LQTS-susceptibility genes that account for nearly $80 \%$ of the disorder (Table 1 ). In addition, three atypical LQTS or multisystem syndromic disorders associated with either QT or QTU prolongation have been described, namely ankyrin B syndrome (formerly LQT4), Andersen-Tawil syndrome (ATS, formerly LQT7), and Timothy syndrome (TS, formerly LQT8).

\section{The Major LQTS Genotypes}

\section{The Big Three: KCNQ1, KCNH2, and SCN5A}

Approximately $75 \%$ of patients with a clinically certain LQTS diagnosis have mutations in one of three major LQTSsusceptibility genes that encode for ion channel $\alpha$ subunits and are critically responsible for the orchestration of the cardiac action potential: KCNQ1-encoded $\mathrm{I}_{\mathrm{Ks}_{\mathrm{s}}}\left(\mathrm{K}_{\mathrm{v}} 7.1\right)$ potassium channel, KCNH2encoded $\mathrm{I}_{\mathrm{Kr}}\left(\mathrm{K}_{\mathrm{v}} 11.1\right)$ potassium channel, or $S C N 5 A$-encoded $\mathrm{I}_{\mathrm{Na}}$ $\left(\mathrm{Na}_{\mathrm{v}} 1.5\right)$ sodium channel. ${ }^{4-6}$ Loss-of-function mutations in $\mathrm{KCNQ1}$ cause about $35 \%$ of LQTS type 1 (LQT1), while loss-of-function KCNH2 mutations contribute approximately $30 \%$ of LQTS (LQT2). Gain-of-function SCN5A mutations underlie roughly 10\% of LQTS (LQT3). About $5 \%$ to $10 \%$ of LQTS patients host multiple mutations in these genes and typically present at a younger age with a more severe phenotype. ${ }^{4}$ The vast majority of mutations are single nucleotide substitutions or small insertion/deletions. ${ }^{4-6}$ However, a few large gene rearrangements resulting in single or multiple whole exon deletions/duplications have been described..$^{7-9}$

Relatively gene-specific triggers, ECG patterns, and therapeutic responses have emerged. ${ }^{10,11}$ For example, while swimming and exertion-induced cardiac events are strongly associated with LQT1, auditory triggers and events occurring during the postpartum period usually occur in patients with LQT2, and events occurring during periods of sleep/rest are most common in LQT3. While exceptions to relatively gene-specific T-wave patterns exist, LQT1 is associated with a broad-based T wave and electrocardiographic features, LQT2 with a low-amplitude notched or biphasic T wave, and LQT3 with a long isoelectric segment followed by a narrow-based T wave. Beta blockers are extremely protective in LQT1 patients but are only moderately protective in LQT2 and LQT3. ${ }^{12,13}$ Female LQT2 patients may not be as fully protected with beta blockers as male LQT2 patients. Given the electrophysiological consequence of an LQT3-causing SCN5A mutation, late sodium current blockers including mexiletine, flecainide, or ranolazine may represent gene-specific therapeutic options for LQT3. ${ }^{14,15}$ However, the response to sodium channel blockers is mutation-specific, and while there has been clear evidence of the benefit of mexiletine in some LQT3 patients, others have shown no benefit. ${ }^{11}$ 


\begin{tabular}{|c|c|c|}
\hline GENE & Locus & PROTEIN \\
\hline \multicolumn{3}{|l|}{ Long QT Syndrome } \\
\hline $\begin{array}{l}\text { Major LQTS Genes } \\
\text { KCNQ1 (LQT1) } \\
\text { KCNH2 (LQT2) } \\
\text { SCN5A (LQT3) }\end{array}$ & $\begin{array}{l}11 p 15.5 \\
7 q 35-36 \\
3 p 21-p 24\end{array}$ & $\begin{array}{l}\mathrm{I}_{\mathrm{Ks}} \text { potassium channel } \alpha \text { subunit }\left(\mathrm{K}_{\mathrm{v}} \mathrm{LQT} 1, \mathrm{~K}_{\mathrm{v}} 7.1\right) \\
\mathrm{I}_{\mathrm{Kr}} \text { potassium channel } \alpha \text { subunit }\left(\mathrm{HERG}, \mathrm{K}_{\mathrm{v}} 11.1\right) \\
\text { Cardiac sodium channel } \alpha \text { subunit }\left(\mathrm{Na}_{\mathrm{v}} 1.5\right)\end{array}$ \\
\hline $\begin{array}{l}\text { Minor LQTS Genes } \\
\text { (listed alphabetically) } \\
\text { AKAP9 } \\
\text { CACNA1C } \\
\text { CALM1 } \\
\text { CALM2 } \\
\text { CAV3 } \\
\text { KCNE1 } \\
\text { KCNE2 } \\
\text { KCNJ5 } \\
\text { SCN4B } \\
\text { SNTA1 }\end{array}$ & $\begin{array}{l}7 \mathrm{q} 21-\mathrm{q} 22 \\
12 \mathrm{p} 13.3 \\
14 \mathrm{q} 32.11 \\
2 \mathrm{p} 21 \\
3 \mathrm{p} 25 \\
21 \mathrm{q} 22.1 \\
21 \mathrm{q} 22.1 \\
11 \mathrm{q} 24.3 \\
11 \mathrm{q} 23.3 \\
20 \mathrm{q} 11.2\end{array}$ & $\begin{array}{l}\text { Yotiao } \\
\text { Voltage gated L-type calcium channel }\left(\mathrm{Ca}_{v} 1.2\right) \\
\text { Calmodulin } \\
\text { Calmodulin } \\
\text { Caveolin-3 } \\
\mathrm{K}_{\mathrm{v}} 7.1 \text { potassium channel beta subunit (MinK) } \\
\mathrm{K}_{\mathrm{v}} 11.1 \text { potassium channel beta subunit (MiRP1) } \\
\text { Potassium inwardly-rectifying channel (Kir3.4) } \\
\text { Sodium channel beta } 4 \text { subunit } \\
\text { Syntrophin-alpha } 1\end{array}$ \\
\hline \multicolumn{3}{|l|}{ Ankyrin-B Syndrome } \\
\hline ANK2 & $4 q 25-q 27$ & Ankyrin B \\
\hline \multicolumn{3}{|l|}{ Andersen-Tawil Syndrome } \\
\hline KCNJ2 (ATS1) & $17 q 23$ & $\mathrm{I}_{\mathrm{K} 1}$ potassium channel (Kir2.1) \\
\hline \multicolumn{3}{|l|}{ Timothy Syndrome } \\
\hline CACNA1C & 12p13.3 & Voltage gated L-type calcium channel (CaV1.2) \\
\hline
\end{tabular}

Table 1. Summary of long QT syndrome-susceptibility genes.

In general, when the QTc is $>500 \mathrm{~ms}$, LQT2 females and LQT3 males are at higher risk for a cardiac event. ${ }^{11}$ In addition, intragenic risk stratification has been realized for LQT1 and LQT2 based upon mutation type, location, and cellular function..$^{16-21}$ LQT1 patients with transmembrane-spanning, domain-localizing KCNQ1 missense mutations and patients with mutations resulting in a greater degree of $\mathrm{K}_{\mathrm{v}} 7.1$ loss-of-function (dominant-negative) are at greater risk of an LQT1-triggered cardiac event compared to LQT1 patients with C-terminal region mutations or those with mutations that cause less damage to the biology of the $K_{v} 7.1$ channel (haploinsufficency), respectively. LQT2 patients with pore region $\mathrm{KCNH} 2$ mutations have a longer QTc, a more severe clinical manifestation of the disorder, and more arrhythmia-related cardiac events occurring at a younger age than those LQT2 patients with non-pore mutations in KCNH2..$^{22}$ In addition, Shimizu et al. found that LQT2 patients with transmembrane pore region mutations had the greatest risk for cardiac events, those with frame-shift/ nonsense mutations in any channel region had an intermediate risk, and those with C-terminus missense mutations had the lowest risk for cardiac events. ${ }^{21}$

\section{The Minor LQTS Genotypes}

The 10 minor LQTS-susceptibility genes encode for additional ion channel $\alpha$ subunits (CACNA1C, KCNJ5), key cardiac potassium-
(AKAP9, KCNE1, KCNE2) and sodium-channel (CAV3, SCN4B SNTA1) interacting proteins, or calcium-binding messenger proteins (CALM1, CALM2). Because these additional genes play a minor role in the genetic basis of LQTS, only limited genotypephenotype correlations have been generated.

\section{CACNA1C-LQTS}

In 2012, Boczek and colleagues used a pedigree-based whole exome sequencing and systems biology strategy to identify a novel pathogenic mutation (P857R) within the CACNA1Cencoded cardiac L-type calcium channel (LTCC) $\alpha$ subunit that cosegregated with disease in a phenotype-positive/genotypenegative multigenerational nonsyndromic LQTS pedigree. ${ }^{23}$ The LTCC is important for excitation-contraction coupling in the heart and mediates an inward depolarizing current in cardiomyocytes. Functional characterization of the mutation using a whole-cell patch-clamp technique in HEK293 cells revealed a gain-of-function with increased $\mathrm{I}_{\mathrm{Ca}, \mathrm{L}}$ and increased cell surface expression of the mutant ion channel compared to wild type. This electrophysiological in vitro phenotype is consistent with the clinical phenotype of QT interval prolongation. Importantly, although CACNA1C perturbations had been implicated previously in Timothy Syndrome (TS) and incorrectly given the genotype place holder as LQT8, this was the first demonstration that 
CACNA1C was a bona fide LQTS-susceptibility gene, extending the breadth of distinct CACNA1C-related arrhythmogenic phenotypes.

Subsequently, mutational analysis of 103 unrelated LQTS genotype-negative/phenotype-positive patients identified three additional $C A C N A 1 C$ missense mutations, suggesting that $C A C N A 1 C$ mutations may explain as much as $4 \%$ to $5 \%$ of genetically elusive LQTS ${ }^{23}$ or approximately $1 \%$ of LQTS altogether. Interestingly, three of the four mutations (K834D, P857L, and P857R) localized to the same critical PEST-domain in the II-III linker of the LTCC, an amino acid sequence motif that represents an important signal for rapid protein degradation and LTCC channel stability. A fourth mutation (R1906C) localizes near the stromal interaction molecule 1 (STIM1) binding domain in LTCC's C-terminus. STIM1 interacts with the LTCC, resulting in a decrease of LTCC-mediated current, and chronically triggers LTCC internalization. Disruption of this STIM1/LTCC interaction could conceivably result in an increase of cell surface expression of the LTCC, thus leading to an overall increase of $\mathrm{I}_{\mathrm{Ca}, \mathrm{L}}$ and subsequently an increase in cardiac action potential duration and a prolonged QT on ECG.

\section{KCNJ5-LQTS}

Yang and colleagues performed a genome-wide linkage and positional candidate gene analysis in a large multigenerational Chinese LQTS pedigree and identified a heterozygote G387R mutation in the KCNJ5-encoded G protein-coupled, inwardly rectifying potassium channel subunit Kir3.4. ${ }^{24}$ In vitro heterologous expression studies revealed a loss-of-function electrophysiological phenotype associated with reduced plasma membrane expression. All mutation-positive family members experienced recurrent palpitations, 10 of 12 with recurrent syncope, and 5 with either persistent or permanent atrial fibrillation (AF) or atrial tachycardia (AT). While the majority of the mutation-positive individuals were symptomatic, only three had a QTc $>480 \mathrm{~ms}$, all with concomitant AF or AT. KCNJ5-mediated LQTS appears to be very uncommon as none of our 500-plus unrelated LQTS probands have been KCNJ5 positive (data not shown).

\section{Channel Interacting Protein-Mediated LQTS}

Importantly, ion channels do not operate in isolation but instead function as macromolecular complexes consisting of the ion channel pore-containing $\alpha$ subunits as well as auxiliary $\beta$ subunits and other regulatory proteins that interact with and influence ion channel activation and deactivation/inactivation. The KCNE1and KCNE2-encoded $\beta$ subunits $\operatorname{minK}$ and MiRP1 were the first auxiliary proteins to be implicated in the pathogenesis of LQTS through their modulatory effect on the $\mathrm{K}_{\mathrm{v}} 7.1\left(K C N Q 1, \mathrm{I}_{\mathrm{Ks}}\right)$ and $\mathrm{K}_{\mathrm{v}} 11.1$ $\left(K C N H 2, \mathrm{I}_{\mathrm{Kr}}\right)$ potassium channels, respectively. ${ }^{25,26}$ The AKAP9encoded yotiao is an A-kinase-anchoring protein that is critically important to the PKA-dependent phosphorylation state of $\mathrm{K}_{\mathrm{v}} 7.1$. In 2007, a single mutation identified in a clinically definite unrelated genotype-negative LQTS patient reduced the interaction between $\mathrm{K}_{\mathrm{v}} 7.1$ and yotiao, eliminated the functional response of the $\mathrm{I}_{\mathrm{Ks}}$ channel to CAMP, and resulted in action potential prolongation in a computational model of the ventricular cardiomyocte. ${ }^{27}$

Similarly, the cardiac sodium channel $\left(\mathrm{Na}_{\mathrm{v}} 1.5\right)$ encoded by $S C N 5 A$ also forms macromolecular complexes with auxiliary proteins. The $S C N 4 B$-encoded $\beta 4$ subunit was implicated in LQTS with the identification of an L179F mutation in a 21-month-old female with intermittent 2:1 atrioventricular block and extreme QT prolongation (QTc, $712 \mathrm{~ms}){ }^{28}$ Coexpression of the L179F-SCN4B mutation with wild-type SCN5A led to a significant increase in persistent late sodium current consistent with an LQT3-like electrophysiological phenotype. However, subsequent mutation analysis of $S C N 4 B$ in a cohort of 262 unrelated genotype-negative LQTS patients failed to identify any additional mutations.

The cardiac sodium channel localizes to omega-shaped membrane microdomains called caveolae. Caveolin-3 encoded by $C A V 3$ is a major scaffolding protein present in caveolae of the heart that may play a role in compartmentalization and regulation of resident ion channels in the caveolae. In 2006, two spontaneous de novo mutations were identified among 905 unrelated LQTS patients referred for genetic testing, thereby demonstrating a pathogenic link between CAV3 mutations and LQTS. ${ }^{29}$ Both CAV3 mutations resulted in a significant LQT3-like increase in persistent late sodium current.

Finally, $\alpha 1$-syntrophin (SNTA1) acts as a molecular scaffold between neuronal nitric oxide synthase (nNOS) and the nNOS inhibitor plasma membrane Ca-ATPase subtype $4 \mathrm{~b}$ (PMCA4b) and interacts with SCN5A to bring the nNOS-PMCA4b complex into close proximity to the cardiac sodium channel. ${ }^{30}$ Additionally, an A390V-SNTA1 mutation identified in a clinically definite, unrelated, genotype-negative LQTS patient disrupted SNTA1 binding with PMCA4b, released inhibition of nNOS, caused S-nitrosylation of SCN5A, and was associated with increased late sodium current. ${ }^{30}$ In a later study, the identical A257G-SNTA1 mutation was identified in 3 of 39 unrelated genotype-negative LQTS cases and also exhibited an in vitro LQT3-like SCN5A gain of function. ${ }^{30,31}$

\section{Calmodulin-Mediated LQTS}

In 2013, a whole exome sequencing-based strategy elucidated the underlying genetic cause for two unrelated sporadic cases of infantile LQTS with recurrent cardiac arrest and extreme QT prolongation. ${ }^{32}$ Both infants hosted sporadic de novo mutations (D130G-CALM1 and D96V-CALM2) in genes (CALM1 and CALM2) that encode for calmodulin, a ubiquitously expressed and essential calcium-signaling protein that is critically involved in a multitude of physiological functions-including as a $\mathrm{Ca}^{2+}$ sensor for $\mathrm{Ca}^{2+}$ dependent inactivation of the LTCC (Cav1.2), inactivation of the cardiac sodium channel (Nav1.5), and activation of the voltagegated potassium channel $\left(\mathrm{K}_{\mathrm{v}} 7.1\right)$. The calmodulin genes represent an interesting and rare phenomenon in human biology. There are three distinct calmodulin genes with distinct loci (CALM1, Chr.14q32.11; CALM2, Chr.2p21; and CALM3, Chr.19q13.2-q13.3); while they share $76 \%$ homology at the DNA nucleotide level, these three genes encode for an identical protein (Calmodulin) of 149 amino acids. All three genes are expressed in cardiac myocytes, with transcript expression levels highest for CALM3 followed by CALM2 and CALM1. ${ }^{32}$

In a subsequent cohort analysis involving 82 unrelated LQTS cases that remained genetically elusive following analysis of the major LQTS genes, calmodulin mutations were identified in two additional cases. ${ }^{32}$ These missense mutations localize to critical EF-hand calcium-binding motifs and reduce calmodulin's calciumbinding affinity by seven-fold. All four calmodulin-positive cases exhibited the common cardiac features of life-threatening ventricular arrhythmias occurring very early in life (three of four during infancy), including frequent T-wave alternans (all cases), markedly prolonged QT intervals (QTc $>600 \mathrm{~ms}$, all cases), and intermittent 2:1 AV block (3 of 4 patients). Ventricular fibrillation was often triggered by adrenergic activation occurring either spontaneously or preceded by a short episode of torsade de pointes 
that was not pulse dependent. Additionally, all patients had some degree of neurodevelopmental delay ranging from mild delay in language development to severe cognitive or motor development. Seizures were observed in three cases.

\section{Multisystem Disorders Associated with Either Prolonged QT or QTU Intervals}

\section{Ankyrin-B Syndrome (formerly LQT4)}

Originally labeled type 4 LQTS (LQT4), this disorder has been renamed more correctly as sick sinus syndrome with bradycardia,

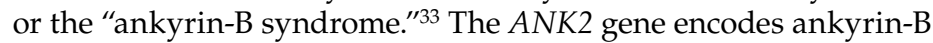
protein, which is involved in anchoring the $\mathrm{Na} / \mathrm{K}-\mathrm{ATPase}, \mathrm{Na} /$ $\mathrm{Ca}$ exchanger, and InsP3 receptor to specialized microdomains in the cardiomyocyte transverse tubules. ${ }^{33}$ Since the discovery of the first human ANK2 mutation identified in a large, multigenerational French pedigree presenting with "atypical LQTS,"33 several lossof-function ankyrin-B mutations have been identified in patients with various arrhythmia phenotypes, including bradycardia, sinus node dysfunction, delayed cardiac conduction/conduction block, idiopathic ventricular fibrillation, AF, drug-induced LQTS, and exercise-induced ventricular tachycardia.

\section{Andersen-Tawil Syndrome (formerly LQT7)}

Andersen-Tawil syndrome (ATS) is a rare multisystem disorder characterized by a triad of clinical features including periodic paralysis, dysmorphic features, and ventricular arrhythmias. ${ }^{34}$ ATS1 was initially coined type 7 LQTS (LQT7) due to the reported observation of extreme prolongation of the QT interval; however, these measurements erroneously included the prominent U-wave. ${ }^{35,36}$ ATS may manifest with pronounced QTU prolongation, prominent U-waves, and ventricular ectopy, including polymorphic ventricular tachycardia (VT), bigeminy, and bidirectional VT.

Mutations in KCNJ2-encoded Kir2.1, a small potassium channel $\alpha$ subunit that is responsible for the inward rectifying cardiac $I_{K 1}$ current that plays an important role in setting the heart's resting membrane potential, accounts for two-thirds of ATS. Most ATS1associated KCNJ2 mutations are missense mutations that cause a loss of function of $\mathrm{I}_{\mathrm{K} 1} \cdot{ }^{35}$ The molecular basis of the remaining third of ATS cases remains genetically and mechanistically elusive.

\section{Timothy Syndrome (formerly LQT8)}

Timothy syndrome (TS) is an extremely rare, multisystem, highly lethal arrhythmia disorder associated with extreme QT prolongation, dysmorphic facial features, congenital heart disease, immune deficiency, developmental delay, and often syndactyly. ${ }^{37}$ Most TS children have potentially fatal arrhythmias including 2:1 atrioventricular block, torsade de pointes, and ventricular fibrillation.

Remarkably, the same recurrent sporadic de novo missense CACNA1C mutation, G406R, in the alternatively spliced exon 8A has been identified in nearly all unrelated TS cases. ${ }^{37}$ In addition, two cases of atypical TS have been described with sporadic de novo CACNA1C mutations not in exon $8 \mathrm{~A}$ but in exon 8 . One case hosted a G406R mutation in exon 8 that was analogous to the classic TS mutation identified in exon $8 \mathrm{a}$. The other case hosted a G402R missense mutation. ${ }^{38}$ These three CACNA1C missense mutations that confer gain-of-function to the LTCC through impaired channel inactivation account for all TS cases analyzed to date. ${ }^{37,38}$

\section{Genetic Testing in Long QT Syndrome}

From a clinical test standpoint, any patient with a strong clinical index of suspicion for a LQTS diagnosis or an asymptomatic patient with an unequivocal prolonged QTc ( $>480 \mathrm{~ms}$ during pre-puberty, > $500 \mathrm{~ms}$ during adulthood) in the absence of other clinical conditions should be offered clinical LQTS genetic testing. ${ }^{39}$ However, genetic tests must be understood as probabilistic rather than unconditionally deterministic, and the genetic test results must be interpreted cautiously and incorporated into the overall diagnostic evaluation for these disorders. 3,39

Conflict of Interest Disclosure: Dr. Ackerman is a consultant for Boston Scientific, Gilead Sciences, Medtronic, and St. Jude Medical.

Funding/Support: Intellectual property derived from Dr. Ackerman's research program resulted in license agreements in 2004 between Mayo Medical Ventures and Genaissance Pharmaceuticals (now Transgenomic), leading to royalties for FAMILION-LQTS and FAMILION-CPVT genetic tests.

Keywords: long QT syndrome; ion channel; genetics

\section{References}

1. Schwartz PJ, Crotti L, Insolia R. Long-QT syndrome: from genetics to management. Circulation. 2012 Aug 1;5(4):868-77.

2. Schulze-Bahr E, Haverkamp W, Wedekind H, Rubie C, Hordt M, Borggrefe M, et al. Autosomal recessive long-QT syndrome (Jervell Lange-Nielsen syndrome) is genetically heterogeneous. Hum Genet. 1997 Oct;100(5-6):573-76.

3. Tester DJ, Ackerman MJ. Genetic testing for potentially lethal, highly treatable inherited cardiomyopathies/channelopathies in clinical practice. Circulation. 2011 Mar 8;123(9):1021-37.

4. Tester DJ, Will ML, Haglund CM, Ackerman MJ. Compendium of cardiac channel mutations in 541 consecutive unrelated patients referred for long QT syndrome genetic testing. Heart Rhythm. 2005 May;2(5):507-17.

5. Splawski I, Shen J, Timothy KW, Lehmann MH, Priori S, Robinson JL, et al. Spectrum of mutations in long-QT syndrome genes. KVLQT1, HERG, SCN5A, KCNE1, and KCNE2. Circulation. 2000 Sep 5;102(10):1178-85.

6. Napolitano C, Priori SG, Schwartz PJ, Bloise R, Ronchetti E, Nastoli J, et al. Genetic testing in the long QT syndrome: development and validation of an efficient approach to genotyping in clinical practice.[See comment.] JAMA. 2005 Dec 21;294(23):2975-80.

7. Tester DJ, Benton AJ, Train L, Deal B, Baudhuin LM, Ackerman MJ. Prevalence and spectrum of large deletions or duplications in the major long QT syndrome-susceptibility genes and implications for long QT syndrome genetic testing. Am J Cardiol. 2010 Oct 15;106(8):1124-28.

8. Koopmann TT, Alders M, Jongbloed RJ, Guerrero S, Mannens MM, Wilde AA, et al. Long QT syndrome caused by a large duplication in the $\mathrm{KCNH} 2$ (HERG) gene undetectable by current polymerase chain reaction-based exon-scanning methodologies. Heart Rhythm. 2006 Jan;3(1):52-5.

9. Eddy CA, MacCormick JM, Chung SK, Crawford JR, Love $\mathrm{DR}$, Rees MI, et al. Identification of large gene deletions and duplications in KCNQ1 and $\mathrm{KCNH} 2$ in patients with long QT syndrome. Heart Rhythm. 2008 Sep;5(9):1275-81.

10. Ackerman MJ. Genotype-phenotype relationships in congenital long QT syndrome. J Electrocardiol. 2005 Oct;38(4 Suppl):64-8.

11. Schwartz PJ, Ackerman MJ, George AL Jr, Wilde AAM. Impact of genetics on the clinical management of channelopathies. J Am Coll Cardiol. 2013 Jul 16;62(3):169-80. 
12. Villain E, Denjoy I, Lupoglazoff JM, Guicheney P, Haingue B, Lucet $\mathrm{V}$, et al. Low incidence of cardiac events with B-blocking therapy in children with long QT syndrome. Eur Heart J. 2004 Aug;25(16):1405-11.

13. Vincent GM, Schwartz PJ, Denjoy I, Swan H, Bithell C, Spazzolini C, et al. High efficacy of beta-blockers in long-QT syndrome type 1: contribution of noncompliance and QT-prolonging drugs to the occurrence of beta-blocker treatment "failures". [See comment.] Circulation. 2009 Jan $20 ; 119(2): 215-21$.

14. Moss AJ, Windle JR, Hall WJ, Zareba W, Robinson JL, McNitt $S$, et al. Safety and efficacy of flecainide in subjects with Long QT-3 syndrome (DeltaKPQ mutation): a randomized, double-blind, placebo-controlled clinical trial. Ann Noninvasive Electrocardiol. 2005 Oct;10(4 Suppl):59-66.

15. Moss AJ, Goldenberg I. Importance of Knowing the Genotype and the Specific Mutation When Managing Patients with Long QT Syndrome.[See comment.] Circulation. Aug 2008;1(3):21326; discussion 226.

16. Moss AJ. T-wave patterns associated with the hereditary long QT syndrome. Card Electrophysiol Rev. 2002 Sep;6(3):311-5.

17. Jons C, Moss AJ, Lopes CM, McNitt S, Zareba W, Goldenberg I, et al. Mutations in conserved amino acids in the KCNQ1 channel and risk of cardiac events in type-1 long-QT syndrome. J Cardiovasc Electrophysiol. 2009 Aug;20(8): 859-65.

18. Shimizu W, Horie M, Ohno S, Takenaka K, Yamaguchi M, Shimizu M, et al. Mutation site-specific differences in arrhythmic risk and sensitivity to sympathetic stimulation in the LQT1 form of congenital long QT syndrome: multicenter study in Japan. J Am Coll Cardiol. 2004 Jul 7;44(1):117-25.

19. Moss AJ, Shimizu W, Wilde AA, Towbin JA, Zareba W, Robinson JL, et al. Clinical aspects of type-1 long-QT syndrome by location, coding type, and biophysical function of mutations involving the KCNQ1 gene. Circulation. 2007 May 15;115(19):2481-9.

20. Nagaoka I, Shimizu W, Itoh H, Yamamoto S, Sakaguchi T, Oka $Y$, et al. Mutation site dependent variability of cardiac events in Japanese LQT2 form of congenital long-QT syndrome. Circ J. 2008 May;72(5):694-9.

21. Shimizu W, Moss AJ, Wilde AA, Towbin JA, Ackerman MJ, January CT, et al. Genotype-phenotype aspects of type 2 long QT syndrome. J Am Coll Cardiol. 2009 Nov 24;54(22):2052-62.

22. Moss AJ, Zareba W, Kaufman ES, Gartman E, Peterson $\mathrm{DR}$, Benhorin J, et al. Increased risk of arrhythmic events in long-QT syndrome with mutations in the pore region of the human ether-a-go-go-related gene potassium channel. Circulation. 2002 Feb 19;105(7):794-9.

23. Boczek NJ, Best JM, Tester DJ, Guidicessi JR, Middha S, Evans JM, et al. Exome sequencing and systems biology converge to identify novel mutations in the L-type calcium channel, CACNA1C, linked to autosomal dominant long QT syndrome. Circ Cardiovasc Genet. 2013 Jun;6(3):279-89.

24. Yang Y, Yang Y, Liang B, Liu J, Li J, Grunnet M, et al. Identification of a Kir3.4 mutation in congenital long QT syndrome. Am J Hum Genet. 2010 Jun 11;86(6):872-80.

25. Splawski I, Tristani-Firouzi M, Lehmann MH, Sanguinetti MC, Keating MT. Mutations in the hminK gene cause long QT syndrome and suppress IKs function. Nat Genet. 1997 Nov;17(3):338-40.
26. Abbott GW, Sesti F, Splawski I, Buck ME, Lehmann MH, Timothy $\mathrm{KW}$, et al. MiRP1 forms IKr potassium channels with HERG and is associated with cardiac arrhythmia. Cell. 1999 Apr 16;97(2):175-87.

27. Chen L, Marquardt ML, Tester DJ, Sampson KJ, Ackerman MJ, Kass RS. Mutation of an A-kinase-anchoring protein causes long-QT syndrome. Proc Natl Acad Sci U S A. 2007 Dec 26;104(52):20990-95

28. Medeiros-Domingo A, Kaku T, Tester DJ, Iturralde-Torres $P$, Itty A, Ye B, et al. SCN4B-encoded sodium channel beta4 subunit in congenital long-QT syndrome. Circulation. $2007 \mathrm{Jul}$ 10;116(2):134-42.

29. Vatta M, Ackerman MJ, Ye B, Makielski JC, Ughanze EE, Taylor EW, et al. Mutant caveolin-3 induces persistent late sodium current and is associated with long-QT syndrome. Circulation. 2006 Nov 14;114(20):2104-12.

30. Ueda K, Valdivia C, Medeiros-Domingo A, Tester DJ, Vatta $M$, Farrugia $G$, et al. Syntrophin mutation associated with long QT syndrome through activation of the nNOS-SCN5A macromolecular complex. Proc Natl Acad Sci U S A. 2008 Jul 8;105(27):9355-60.

31. Wu G, Ai T, Kim JJ, Mohapatra B, Xi Y, Li Z, et al. alpha-1syntrophin mutation and the long-QT syndrome: a disease of sodium channel disruption. Circ Arrhythm Electrophysiol. 2008 Aug;1(3):193-201.

32. Crotti L, Johnson CN, Graf E, De Ferrari GM, Cuneo BF, Ovadia M, et al. Calmodulin mutations associated with recurrent cardiac arrest in infants. Circulation. 2013 Mar 5;127(9):1009-17.

33. Mohler PJ, Le Scouarnec S, Denjoy I, Lowe JS, Guicheney P Caron $L$, et al. Defining the cellular phenotype of "ankyrin-B syndrome" variants: human ANK2 variants associated with clinical phenotypes display a spectrum of activities in cardiomyocytes. Circulation. 2007 Jan 30;115(4):432-41.

34. Yoon G, Oberoi S, Tristani-Firouzi M, Etheridge SP, Quitania $\mathrm{L}$, Kramer JH, et al. Andersen-Tawil syndrome: prospective cohort analysis and expansion of the phenotype. Am J Med Genet A. 2006 Feb 15;140(4):312-21.

35. Tristani-Firouzi M, Jensen JL, Donaldson MR, Sansone V, Meola G, Hahn A, et al. Functional and clinical characterization of KCNJ2 mutations associated with LQT7 (Andersen syndrome). J Clin Invest. 2002 Aug 1;110(3):381-8.

36. Zhang L, Benson DW, Tristani-Firouzi M, Ptacek LJ, Tawil R, Schwartz PJ, et al. Electrocardiographic features in AndersenTawil syndrome patients with KCNJ2 mutations: characteristic $\mathrm{T}$-U-wave patterns predict the KCNJ2 genotype. Circulation. 2005 May 31;111(21):2720-6.

37. Splawski I, Timothy KW, Sharpe LM, Decher N, Kumar P, Bloise $R$, et al. $\mathrm{Ca}(\mathrm{V}) 1.2$ calcium channel dysfunction causes a multisystem disorder including arrhythmia and autism. Cell. 2004 Oct 1;119(1):19-31.

38. Splawski I, Timothy KW, Decher N, Kumar P, Sachse FB, Beggs $\mathrm{AH}$, et al. Severe arrhythmia disorder caused by cardiac L-type calcium channel mutations. Proc Natl Acad Sci U S A. 2005 Jun 7;102(23):8089-96.

39. Ackerman MJ, Priori SG, Willems S, Berul C, Brugada R, Calkins $\mathrm{H}$, et al. HRS/EHRA expert consensus statement on the state of genetic testing for the channelopathies and cardiomyopathies this document was developed as a partnership between the Heart Rhythm Society (HRS) and the European Heart Rhythm Association (EHRA). Heart Rhythm. 2011 Aug;8(8):1308-39. 